\title{
The use of mathematical modeling methods for solving geo- ecological problems of sustainable development of regions
}

\author{
$M V \operatorname{Volik}^{1,2, *}$ \\ ${ }^{1}$ Department of mathematical modeling, SMI VSC RAS, Vladikavkaz, Russia \\ ${ }^{2}$ Department of mathematics and computer science, Financial University, Vladikavkaz, Russia
}

\begin{abstract}
Currently, a number of environmental problems have a significant impact on the stable economic development of the country. A global problem is the study of air pollution. The solution of such geoecological problems should be carried out with the use of modern mathematical apparatus and digital technologies. The paper presents the results of a numerical study of the distribution of gaseous pollutants emitted by motor vehicles in the pedestrian zone of streets. It is shown that the vortex structures formed in the studied city buildings development have a significant impact on the accumulation of anthropogenic impurities.
\end{abstract}

\section{Introduction}

Currently, environmental problems remain relevant throughout the world despite a number of activities aimed not only at solving the problems that have arisen, but also at predicting and preventing them. It is obvious that all these measures require financial investments and have a significant impact on the economy of each country. The introduction and use of environmentally friendly technologies depends on many factors (scale, income, company expenses, etc.). There is greening of modern society everywhere, which is reflected in science, technology, manufacturing, economics, medicine, and other areas of daily life and professional activity. In the development of civilization, such a scientific direction as geo-ecology remains a priority. Studies by Russian and foreign scientists highlight a number of issues that show the current importance of solving geo-ecological problems for the sustainable development of countries and regions, associated with the continuation of environmental deterioration and natural life support systems, as well as the emergence of negative trends in economic and social development. [1] The harmonious development of society in the future is impossible without a mass geo-ecological orientation in various areas of life (from public consciousness to the economy). Each person should understand that it is unacceptable to live only for today, and it is necessary to think about the future of the descendants and their environment. To solve geo-ecological problems it is necessary to carry out complexes of thought-out and strategically grounded measures for decades. Effective control and regulation of the state of the environment is possible only if humanity constantly accumulates reliable data on the current state of the environment, correctly applies the knowledge gained and prevents the damage caused by society. [1-4]

A separate direction of geo-ecology is the study of such a type of connections in which gradual impacts on a system lead to a gradual accumulation of changes in its state. An example of such a phenomenon is atmospheric pollution, which occurs gradually, but after several decades of air quality in settlements is significantly deteriorating. Currently, the problem of air pollution is one of the most difficult. Everyone knows global environmental problems: acid rain, destruction of the ozone layer, the greenhouse effect. Atmospheric air plays a key role among other components of the biosphere, fulfilling its ecological function, protecting the planet from absolutely cold outer space and solar radiation. In the atmosphere global processes related to meteorology, climate and weather occur. By itself, atmospheric air is capable of self-cleaning during leaching of aerosol components by sediments, turbulent movement of the surface layer, distribution of pollutants on the surface, etc. However, modern civilization causes irreparable harm to natural systems that promote selfpurification of the atmosphere. [5-7] In addition, an analysis of the incidence of the population of the regions shows that the human impact on the atmosphere has a very negative impact on human health, especially in cities with developed industry and road infrastructure. [7]

Thus, anthropogenic pollution of atmospheric air has a destructive effect on the ecological state of the environment, which is advisable to constantly monitor, model and predict. In this regard, increases the relevance of monitoring the state of atmospheric air. Theoretical studies on urban ecology, ecological climatology and environmental monitoring were regularly conducted by Russian and foreign scientists. The main approaches to

\footnotetext{
* Corresponding author: volikmv@mail.ru
} 
solving geoecological problems are substantiated in the works of K.S. Bushtueva, E.Yu. Bezugla, ME Berland, Yu.A. Israel, A.A. Isaev, I. Turiel, R. Wilson and others. [8]

\section{Research methods}

For effective environmental protection measures, quality control of the state of emissions to the environment from various sources of pollution is necessary. For example, the mass fraction of emissions from motor vehicles increases every year due to an increase in the number of vehicles. This contributes to the fact that gaseous emissions from cars become the main source of pollution of the urban atmosphere. However, pollutants entering the streets during industrial emissions cannot be ignored. An effective tool for solving environmental problems and a method of ensuring environmental safety is mathematical modeling - the most promising direction in forecasting opportunities, as well as saving material costs and safety of experiments. The tasks of mathematical modeling require a large amount of computer time. One way to overcome this problem is to use multiprocessor computing systems and free software.

Modeling in atmospheric aerodynamics has been developing for a long time. The initiators are considered to be Marchuk G.I., Dorodnitsyn A.A. Mathematical modeling of aerodynamics of city buildings and the spread of pollutants in it continues to develop in Russia (Arguchintseva, AV, Gavrilov, AS, Guvernyuk, SV, Setukha, AV, Sukhinov, AI, Chistyakov, A.E., Starchenko, A.V., Naats, V.Yu. and etc.) and abroad (Hunter I.J., Watson I.D., Johnson G.T., Liu C.H., Barth M.C., Leung D.Y.C., Kovar-Panskus A., Louka P., Sini J-F., Savory E., Czech M., Abdelqar A., Mestayer P.G., Toy N. S. Thangam, M.W.Rotach, M.Pavaro, M. Shatzmann, M.Leitl, P.Kastner-Klein, E.J.Plate and etc.). Models of various dimensions and with varying degrees of detail of physical processes have been developed. [1$18]$

The purpose of this work is to study the aerodynamics of city buildings, the analysis of the distribution of gaseous pollutants inside the streets based on the results of mathematical modeling. To solve this problem, it is actual to use modern methods and algorithms of mathematical modeling, information technology and free software. Mathematical modeling methods are a modern tool for preliminary assessment of the pattern of air flow and the spread of pollutants in in urban setting, facilitating the planning and implementation of measures to prevent environmental problems. [19] In this paper, mathematical modeling of the air flow and the spread of gaseous pollutants was carried out using the open source OpenFoam package and remote access to the resources of the Institute for System Programming named after V.P. Ivannikova RAS (Moscow) - UniHUB Web Labs (www.unihub.ru) under the program «University Cluster» (www.unicluster.ru). [19] The problem solved in OpenFoam contains the initial and boundary conditions, the computational grid, as well as the physical properties and integration parameters of the equations, which are discussed in detail in the papers [19-24]. To carry out computational experiments, the standard pimpleFoam solver was used for turbulent fluid flow, in which the Pimple velocity and pressure coupling algorithm is used. It was assumed that the moving air is incompressible fluid. The K-epsilon turbulence model was used. Computational experiments were carried out in a two-dimensional formulation for the time interval from 0 to $1500 \mathrm{~s}$. in increments of $0.001 \mathrm{~s}$. A uniform computational grid in a rectangular computational domain with a step of $1 \mathrm{~m}$ in space was used, imitating a urban buildings located on the outskirts of the city. The urban development consists of seven parallel streets, with houses $15 \mathrm{~m}$ high and $15 \mathrm{~m}$ wide. The air flow was simulated from left to right. The distance from the entrance boundary to the windward side of the first obstacle was assumed to be ten its heights, from the leeward side of the last obstacle to the exit boundary - twenty, and from the lower boundary of the computational domain to the upper boundary - five heights. Researcheds the options with the location of sources of pollutants inside one of the seven simulated streets.

\section{Research results}

The papers [22-23] present a picture of the flow of air and the spread of gaseous pollutants for the case when sources with the same amount of emissions are located inside each of the seven streets contemporaneously. The results of calculations showed that a multi-vortex structure is formed above the buildings, the horizontal size of which is approximately equal to the four heights of the houses. If the first streets are under the influence of this vortex structure, the air velocity in them turns out to be significantly less, which prevents the ventilation of these streets. Also on the pattern of air flow depends the distribution of pollutants emitted to other streets. In this regard, it is advisable to conduct a numerical simulation of the distribution of gaseous pollutants emitted by sources located inside one of seven streets. The results can be useful from the point of view of regulating the flow of vehicles, namely, limiting the number of cars in some streets.

The Figure 1 shows the distribution of the concentration of pollutants across the width of streets at a height of $2 \mathrm{~m}$ from the lower boundary of the computational area for cases where the source is located

only inside one of seven streets (curves 1 - 7).

It can be seen that the largest amount of pollutants accumulates inside the first and third streets, and there is also a transfer of pollutants to neighboring streets. Thus, it is necessary to conduct a study on the distribution of gaseous pollutants in cases where the sources of pollutants are active inside all streets except the first and/or third.

As a result of calculations, it was found that the vortex structures observed in a computational experiment and the location of sources of pollutants have a significant effect on the concentration of pollutants at the level of pedestrians. Elimination of pollution sources 


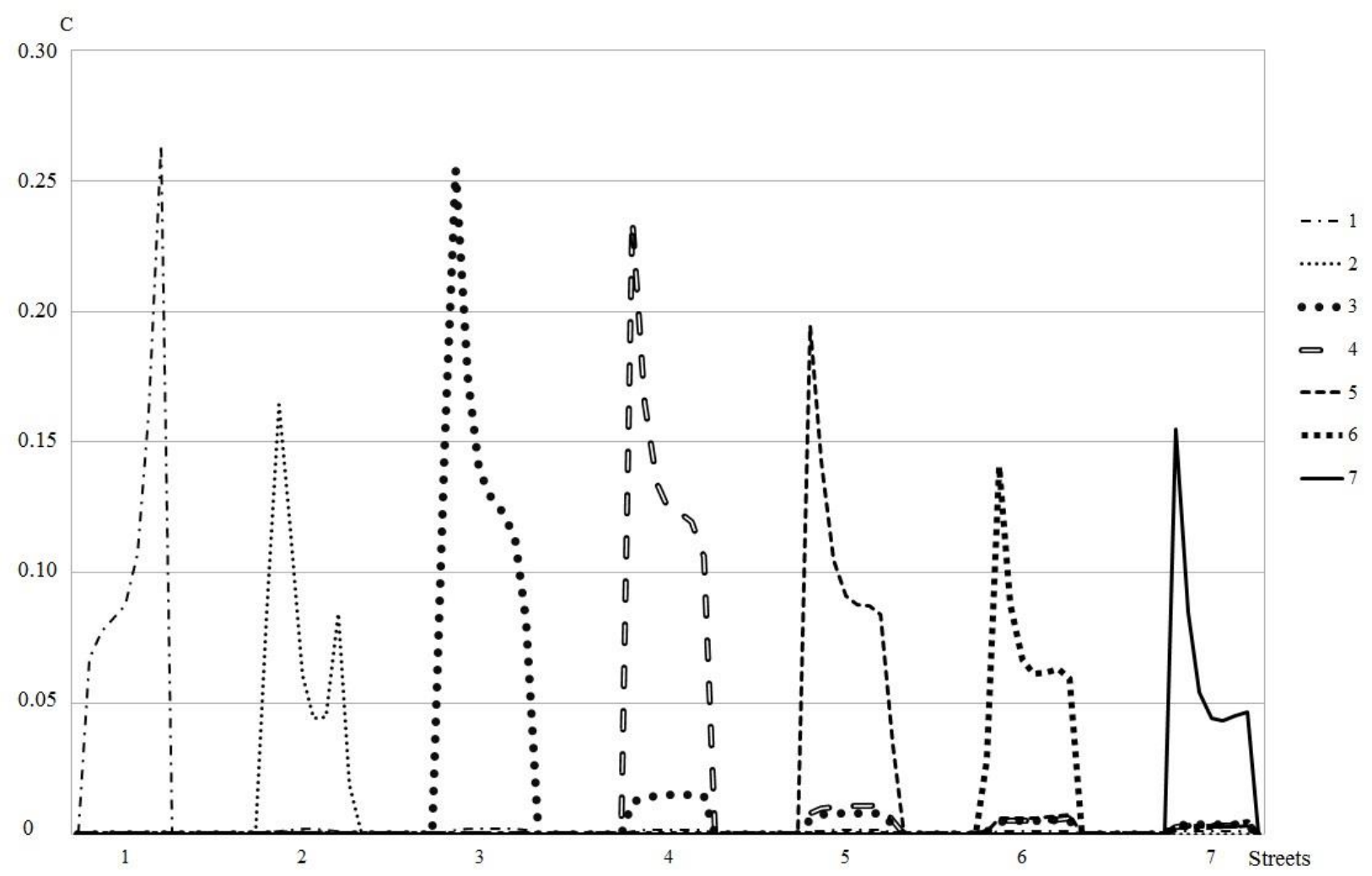

Fig. 1. The distribution of the concentration of gaseous pollutants from motor vehicles across the width of streets at a height of $2 \mathrm{~m}$ from the lower boundary of the computational domain at a time point of 1500 seconds.

in different streets can lead to a decrease in the concentration of pollutants in the pedestrian zone. Further research is advisable to carry out cases of eliminating sources of pollutants, as well as reducing the amount of harmful emissions by reducing the number of vehicles. This will allow identifying areas for carrying out appropriate measurements and preparing recommendations for regulating the flow of vehicles.

\section{Conclusion}

Unfortunately, the global geoecological crisis is becoming more and more frequent, causing fears and concern of the leadership of many countries. The problems of geo-ecology are increasingly attracting the attention of society and raise the level of understanding of the interdisciplinarity of the main global tasks of a systemic nature as a result of the interaction of complex systems. Geoecological tasks are a combination of social, natural, political and economic problems. The leadership of the countries carried out a number of activities aimed at improving the environmental situation. For example, organizational activities urges for the population to careful respect the natural system. In addition, everyone is aware of measures to improve the processing of solid household waste and environmentally friendly production. However, the situation remains tense and requires serious attention and modern scientific research. Due to the fact that not all studies can be carried out in real physical conditions, forecasting environmental situations using mathematical models and digital technologies is becoming increasingly popular among Russian and foreign scientists. In this paper, studied the spread of gaseous pollutants from cars in the pedestrian zone inside city developments. The reliability of the results obtained is due to the correctness of the problem statement using the hydrodynamic equations tested for solving various problems of physics and aerodynamics of the atmosphere, as well as preliminary comparison with the results of observations.

Thus, the use of modern methods of mathematical modeling and information technologies to solve geoecological problems will reduce the costs of measuring, analyzing, modeling and forecasting the ecological situation in the country and the world, as well as contribute to the sustainable development of environmental management.

\section{References}

[1] V.V. Menshikov, Risk analysis of systematic air pollution with hazardous chemicals: a tutoriall, $\mathrm{M}$ $\therefore$ Izd-vo Khimich. fact Mosk. University, 120 (2003).

[2] V.K. Arguchintsev, Assessment of air pollution in Ulan Bator by motor transport, Proceedings of the Irkutsk State University, Series: Earth Sciences, 8, 15-24 (2014).

[3] S.V. Governyuk, O.O. Yegorychev, S.A. Isaev, N.V. Kornev, O.I. Poddaeva, Numerical and 
physical modeling of wind impact on a group of high-rise buildings, Vestnik MGSU, 3, 1, 185-191 (2011).

[4] L. Xian-Xiang, D.Y.C. Leung, L. Chun-ho, K.M. Lam, Physical modeling of flow field inside urban street canyons, Journal of Applied Meteorology And Climatology, 47, 2058-2067 (2008).

[5] M.E. Baranova, A.S. Gavrilov, Methods of calculated monitoring of atmospheric pollution in megacities, Natural and Technical Sciences, 4, 36, 211-225 (2008).

[6] O.V. Kondrakov, O.V. Kryuchin, M.Yu. Volosatov, S.Yu. Kletrov, Modeling the spread of pollutants in the atmosphere on the basis of the "torch" model, Bulletin of the Tambov University. Series: Natural and Technical Sciences, 1 (2011) [Electronic resource]. Available at: http://cyberleninka.ru/article/.

[7] V.I. Naac, I.E. Naac, Mathematical models and numerical methods in problems of environmental monitoring of the atmosphere, M. Fizmatlit, 328, 2010.

[8] A.I. Sukhinov, A.E. Chistyakov, D.S. Khachunts, Mathematical modeling of the movement of multicomponent air and transport of pollutants, Proceedings of the Southern Federal University, Theoretical and applied questions of mathematical modeling, 8, 121, 73-79 (2011).

[9] A.V. Starchenko, R.B. Nuterman, E.A. Danilkin, Numerical modeling of turbulent flows and admixture transport in street canyons, Tomsk: Publishing house Tom. University, 252 (2015).

[10] P. Kastner-Klein, E. Fedorovich, M.W. Rotach, A wind tunnel study of organised and turbulent air motions in urban street canyons, Journal of Wind Engineering and Industrial Aerodynamics, 89, 9, 849-861 (2001).

[11] A. Kovar-Panskus, A. Covar-Panskus, P. Louka, J-F. Sini, E. Savory, M. Czech, A. Abdelqari, P.G. Mestayer, N. Toy, Influence of geometry on the flow and turbulence characteristics within urban street canyons - Intercomparison of wind tunnel experiments and numerical simulations, Proceedings of the 3rd International Conference on Urban Air Quality, Loutraki, Greece (2001) [Electronic resource]. Available at: http: //www.dmu.dk/AtmosphericEnvironment/trapos.

[12] A. Kovar-Panskus, P. Louka, J-F. Sini, E. Savory, M. Czech, A. Abdelqari, P.G. Mestayer, N. Toy, Influence of geometry on the mean flow within urban street canyons - a comparison of wind tunnel experiments and numerical simulations, Journal of Water, Air and Soil Pollution, 2, 365380 (2002).

[13] C.H. Liu, M.C. Barth, D.Y.C. Leung, Large-Eddy Simulation of Flow and Pollutant Transport in Street Canyons of Different Building-Heigth-toStreet-Width Ratios, Journal of Applied Meteorology, 43, 1410-1422 (2004).

[14] G.L. Mellor, T. Yamada, A hierarchi of turbulence closure models for planetary boundary layers, Journal of the atmospheric sciences, 31, 1791-1806 (1974).

[15] M. Pavageau, M. Schatzmann, Wind tunnel measurement of concentration fluctuations in an urban street canyon, Atmospheric Environment, 33, 3961-3971 (1999).

[16] M.W. Rotach, Profiles of turbulence statistics in and above an urban street canyon, Atmospheric Environment, 29 (12), 1473-1486.

[17] M. Schatzmann, M. Leit, Validation and application of obstacle-resolving urban dispersion models, Atmospheric Environment, 36, 48114821 (2002).

[18] K. Uehara, S. Murakami, S. Oikava, S. Wakamatsu, Wind tunnel experiments on how thermal stratification affects flow in and above urban street canyon, Atmospheric Environment, 34, 1533-1562 (2000).

[19] M.V. Kraposhin, O.I. Samovarov, S.V. Strizhak, OpenFoam package: numerical simulation of MSS problems, Materials of the school-seminar "Basics of using OpenFoam, Salome, ParaView" (2014) [Electronic resource]. Available at: https://unihub.ru/tools.

[20] M.V. Volik, N.S. Orlova, Modeling the spread of pollutants emitted from low-lying sources, Modeling of non-equilibrium systems: Proceedings of the XVII All-Russian seminar, Krasnoyarsk: Institute of Computational Modeling, Siberian Branch of the Russian Academy of Sciences, 32-35 (2014).

[21] M.V. Volik, Numerical study of the influence of the width of the obstacle on the spread of pollutants after the elimination of the source of pollution, Proceedings of the KabardinoBalkarian Scientific Center of the Russian Academy of Sciences, 6, 86, 15-21 (2018).

[22] M.V. Volik, Numerical modeling of the spread of pollutants emitted from low-lying sources inside the streets, Proceedings of the KabardinoBalkarian Scientific Center of the Russian Academy of Sciences, 1, 69, 20-27 (2016).

[23] E.S. Kamenetsky, M.V. Volik, A.M. Tagirov, Mathematical modeling of the spread of pollutants emitted by motor transport, Proceedings of the Kabardino-Balkarian Scientific Center of the Russian Academy of Sciences, 6, 62, 23-31 (2014).

[24] N.S. Orlova, M.V. Volik, Simulation of air movement in street canyons using the OpenFOAM package, Processes in geomedia, 2, 11, 529-536 (2017). 